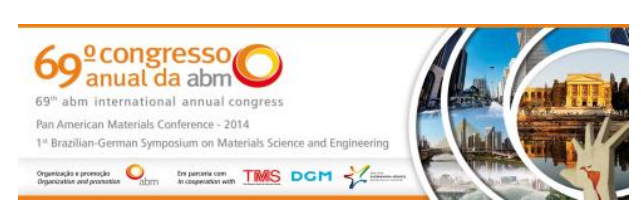

Tema: Materiais com efeito de memória de forma

\title{
FLEXIBILIDADE E RESISTÊNCIA TORCIONAL DE INSTRUMENTOS ROTATÓRIOS DE NITI COM MEMÓRIA DE FORMA*
}

\author{
Lígia Carolina Moreira Braga ${ }^{1}$ \\ Maria Guiomar de Azevedo Bahia² \\ Vicente Tadeu Lopes Buono ${ }^{3}$
}

\section{Resumo}

Este trabalho teve como objetivo avaliar o uso da tecnologia Controlled Memory (CM) para confecção de instrumentos endodônticos rotatórios de NiTi, comparando a flexibilidade e a resistência torcional de instrumentos Hyflex (HF) e Typhoon (TYP), que apresentam o efeito memória de forma, com os instrumentos ProTaper Universal F2 (PTU F2) e EndoWave (EW), fabricados com o fio de NiTi superelástico convencional. Os instrumentos avaliados possuíam calibre de ponta de 0,30 $\mathrm{mm}$ e conicidade de $6 \%$, com exceção de PTU F2. As áreas médias das seções transversais a $3 \mathrm{~mm}$ da ponta (A3) foram determinadas em 10 instrumentos de cada tipo. Igual número de instrumentos foram testados em dobramento a $45^{\circ}$ e em torção até a ruptura (ISO 3630-1). Os valores médios de A3 encontrados nos instrumentos HF, TYP, PTU F2 e EW foram, respectivamente, 0,084, 0,107, 0,122 e 0,082 mm2; os valores médios de momento de dobramento máximo: 0,327, 0,315, 1,1490 e 0,567 N.cm; e os valores médios de torque máximo: 1,047, 1,254, 1,400 e 1,111 N.cm. Verificouse, portanto, que os instrumentos fabricados com a tecnologia CM apresentaram, quando comparados aos instrumentos com valores próximos de A3, maior flexibilidade (menor momento de dobramento máximo) e resistência torcional semelhante. Os resultados encontrados mostraram que a tecnologia $\mathrm{CM}$ permite a produção de instrumentos endodônticos mais flexíveis, o que é desejável do ponto de vista clínico, porém sem comprometer a sua resistência torcional.

Palavras-chave: Ligas NiTi; Efeito memória de forma; Instrumentos endodônticos; Flexibilidade; Resistência torcional.

\section{FLEXIBILITY AND TORSIONAL RESISTANCE OF NITI ROTARY INSTRUMENTS WITH SHAPE MEMORY}

\section{Abstract}

This study aimed to evaluate the use of Controlled Memory (CM) technology for the manufacture of rotary endodontic NiTi instruments, comparing the flexibility and torsional resistance of Hyflex (HF) and Typhoon (TYP) instruments, which exhibit the shape memory effect, with instruments ProTaper Universal F2 (PTU F2) and EndoWave (EW), manufactured with conventional superelastic NiTi wire. The instruments evaluated possessed a $0.30 \mathrm{~mm}$ caliber tip and $6 \%$ taper, except for PTU F2. The mean cross-sectional areas at $3 \mathrm{~mm}$ from the tip (A3) were determined in 10 sections of each instruments type. Equal number of instruments was tested in bending at $45^{\circ}$ and in torsional (ISO 3630-1). The average values of A3 found for HF, TYP, PTU F2 and EW instruments were $0.084,0.107,0.122$ and $0.082 \mathrm{~mm}^{2}$, respectively, while the average values of maximum bending moment were $0.327,0.315,1.1490$ and $0.567 \mathrm{~N} . \mathrm{cm}$, and the mean values of maximum torque: $1.047,1.254,1.400$ and $1.111 \mathrm{~N} . \mathrm{cm}$. These results showed that the $\mathrm{CM}$ technology allows the production of instruments which show, when compared instruments with similar values of $\mathrm{A} 3$, greater flexibility (lowest maximum bending moment) and similar torsional. The results obtained demonstrated that the CM technology enables the production of more flexible endodontic instruments, which is desirable from a clinical point of view, but without compromising their torsional strength.

Keywords: NiTi alloys; Shape memory effect; Endodontic instruments; Flexibility; Torsional strength.

1 Mestre, Doutoranda em Odontologia/Endodontia, Depto. de Odontologia Restauradora, Faculdade de Odontologia, Universidade Federal de Minas Gerais (UFMG), Belo Horizonte, MG, Brasil.

2 Mestre Odontologia/Endodontia, Doutorado em Engenharia Metalúrgica e de Minas, Professora Associada, Depto. de Odontologia Restauradora, Faculdade de Odontologia, UFMG, Belo Horizonte, MG, Brasil.

3 Mestre em Física, Doutorado, Engenharia Metalúrgica e de Minas, Professor Associado IV, Depto. de Engenharia Metalúrgica e de Materiais, UFMG, Belo Horizonte, MG, Brasil.

\footnotetext{
* Contribuição técnica ao 69 Congresso Anual da ABM - Internacional e ao 14ํㅡㄹ ENEMET - Encontro Nacional de Estudantes de Engenharia Metalúrgica, de Materiais e de Minas, 21 a 25 de julho de 2014, São Paulo, SP, Brasil.
} 


\section{INTRODUÇÃO}

A tecnologia da liga níquel-titânio (NiTi) foi introduzida na clínica odontológica há quase duas décadas. Instrumentos endodônticos rotatórios fabricados a partir desta liga foram um grande avanço trazido por causa de suas propriedades únicas de superelasticidade e efeito memória de forma [1], tornando os instrumentos de NiTi mais flexíveis e resistentes à fratura torcional, quando comparados com os instrumentos tradicionais fabricados em aço inoxidável [2].

Durante os últimos 10 anos, ocorreram melhorias significativas na concepção e controle das matérias-primas em relação à microestrutura, as propriedades dos materiais e processos de fabricação de instrumentos endodônticos [3-6]. Melhorias nessas áreas de materiais levaram ao desenvolvimento de novos instrumentos endodônticos, fabricados com um fio de NiTi submetido a um tratamento termomecânico especial e denominado M-Wire, com maior flexibilidade e resistência à fadiga [7-9]. Embora estes instrumentos, recentemente desenvolvidos, têm sido clinicamente bem sucedidos, os fabricantes estão constantemente tentando melhorar ainda mais esses dispositivos. A tecnologia CM (Controlled Memory) é uma nova concepção em que tratamentos térmicos são aplicados ao instrumento já usinado, e não previamente ao fio usado em sua fabricação. De acordo com os fabricantes desses instrumentos, a "memória controlada" permite que canais radiculares complexos sejam instrumentados, minimizando as forças laterais indesejadas que podem transportar e formar degraus nos canais. Além disso, os instrumentos $\mathrm{CM}$ são comercializados como possuindo maior resistência à fadiga e à torção [10]. No entanto, com instrumentos mais avançados de NiTi sendo desenvolvidos e produzidos por diferentes fabricantes, a compreensão da natureza das diferentes matérias-primas e seu impacto no desempenho do instrumento tornou-se mais imperativo para o clínico no que se refere à escolha do instrumento e ao sucesso do tratamento.

Instrumentos fabricados com a tecnologia CM têm se mostrado de $300 \%$ a $800 \%$ mais resistentes à fadiga em comparação aos instrumentos fabricados com o fio de $\mathrm{NiTi}$ convencional [11-14]. No entanto, as propriedades de flexão e torção desses instrumentos foram relatadas apenas em alguns estudos, que possuem resultados contraditórios. Instrumentos CM avaliados comparativamente $[15,16]$ mostraram um aumento de flexibilidade em relação aos de NiTi convencional, mas apresentaram variabilidade em valores do torque máximo entre instrumentos de diferentes fabricantes. Outro estudo constatou que os instrumentos CM apresentaram alta resistência à torção, além de elevada deformação plástica antes de fraturar [17]. De um modo geral, observa-se que não houve padronização de fatores geométricos e dimensionais dos instrumentos nesses trabalhos, o que pode ter afetado os resultados, daí a necessidade de se realizar este novo estudo.

Portanto, o objetivo do presente trabalho foi realizar uma avaliação mais cuidadosa das propriedades mecânicas de instrumentos com a tecnologia CM tomando por base instrumentos com características geométricas semelhantes, porém fabricados utilizando fios de NiTi superelástico convencionais. A informação comparativa deste estudo se destina a ajudar os dentistas a entenderem melhor as diferenças entre os diversos materiais e facilitar boas escolhas clínicas para atender aos desafios anatômicos dos canais radiculares.

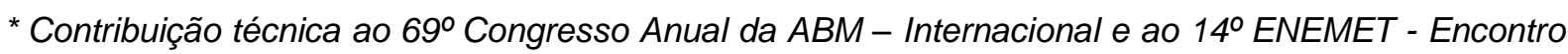
Nacional de Estudantes de Engenharia Metalúrgica, de Materiais e de Minas, 21 a 25 de julho de 2014, São Paulo, SP, Brasil.
} 


\section{MATERIAIS E MÉTODOS}

Instrumentos novos 30/.06 (diâmetro de ponta igual a 0,3 mm e conicidade de 6\%) EndoWave (EW; J. Morita Corporation, Osaka, JP), HyFlex (HF; Coltene/Whaledent, Inc., Cuyahoga Falls, OH), Typhoon (TYP; Clinician's Choice Dental Products, New Milford, CT),) e ProTaper Universal F2 (PTU F2; Dentsply Maillefer, Ballaigues, Switzerland) foram avaliados. Estes instrumentos foram escolhidos porque possuem características geométricas semelhantes: EW e HF apresentam seções transversais triangulares, mas PTU F2 e TYP apresentam seções triangulares convexas. Os instrumentos EW e PTU F2 são fabricados com fios de NiTi superelásticos, enquanto que HF e TYP são produzidos utilizando a tecnologia CM.

Antes dos ensaios mecânicos, dez instrumentos de cada sistema foram fotografados com uma câmera digital de alta resolução (20D; Canon, Tóquio, Japão) para avaliar suas características dimensionais com base na American National Standards Institute/American Dental Association Specification No. 101. Linhas foram desenhadas nas imagens do instrumento, e o comprimento de pitchs (passos da hélice) foi medido usando o programa Image Pro Plus 6.0 (Media Cybernetics, Silver Spring, MD). Os instrumentos, três de cada um dos tamanhos e conicidades avaliados, foram cortados em cerca de $2,7 \mathrm{~mm}$ a partir da ponta com um cortador metalográfico (Isomet 1000; Buehler, Lake Bluff, IL, EUA). As superfícies transversais foram polidas com lixa para chegar a $3,0 \mathrm{~mm}$ da ponta e depois fotografadas com um aumento de 150x no microscópio eletrônico de varredura (MEV: JSM 6360, Jeol, Tóquio, Japão). As áreas da secção transversal foram determinadas usando o mesmo software anteriormente descrito.

Medições de dureza utilizando a técnica de microindentação Vickers (Durimet 2; Leica, Wetzlar, Alemanha), com uma carga de $100 \mathrm{gf}$, foram realizadas em três amostras de cada tipo de instrumento previamente montadas em resina metalográfica, lixadas e polidas com pasta de diamante. Dez indentações foram feitas em cada amostra, totalizando 30 medições por tipo de instrumento.

Dez instrumentos de cada tipo foram utilizados nos ensaios de flexão de acordo com a especificação ISO 3630-1. As condições de ensaio foram descritas em detalhes em [18] e incluem a fixação do instrumento a $3 \mathrm{~mm}$ da ponta e em uma garra de latão e a aplicação de força perpendicular ao longo eixo do instrumento a uma distância de $23 \mathrm{~mm}$ da ponta. A força atua até que o dobramento atinja $45^{\circ}$ enquanto a força é aplicada continuamente. O momento de dobramento (MD) é obtido multiplicando, pelo comprimento do braço de alavanca, a força determinada por uma célula de carga. $O$ momento de dobramento é registrado continuamente pelo equipamento e seu valor correspondente ao dobramento de $45^{\circ}$ é o parâmetro utilizado para avaliação da flexibilidade do instrumento, de acordo com a norma ISO 3630-1.

Outro grupo de instrumentos foi testado em torção até a fratura com base na especificação ISO 3630-1 usando um dispositivo de teste de torção de bancada no qual o instrumento é fixado a $3 \mathrm{~mm}$ da ponta em um mandril de aço com buchas de cobre recozido. O cabo do instrumento é retirado e a haste é fixada na garra da máquina, também utilizando buchas de cobre. O instrumento é então girado sobre seu longo eixo a uma velocidade angular de $2 \mathrm{rpm}$. A posição angular e o torque necessário para girar 0 instrumento sobre sua ponta fixa são registrados continuamente até a ruptura do instrumento, que ocorre no ponto de aprisionamento da ponta.

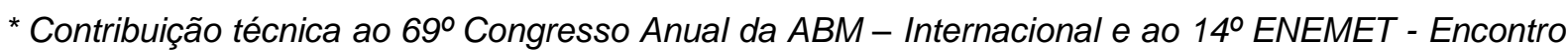
Nacional de Estudantes de Engenharia Metalúrgica, de Materiais e de Minas, 21 a 25 de julho de 2014, São Paulo, SP, Brasil.
} 
A significância estatística das diferenças nos parâmetros medidos entre os diferentes tipos de instrumentos foi determinada por meio de análise de variância one-way em um nível de confiança de $95 \%$.

\section{RESULTADOS E DISCUSSÃO}

O comprimento de pitch aumentou ao longo da parte ativa dos instrumentos EW, PTU F2 e TYP, sendo este aumento mais acentuado nos instrumentos EW (Figura1). Por outro lado, os instrumentos HF mostraram comprimento de pitch constante. Em geral, EW apresentou comprimentos de pitch maiores do que os outros instrumentos.

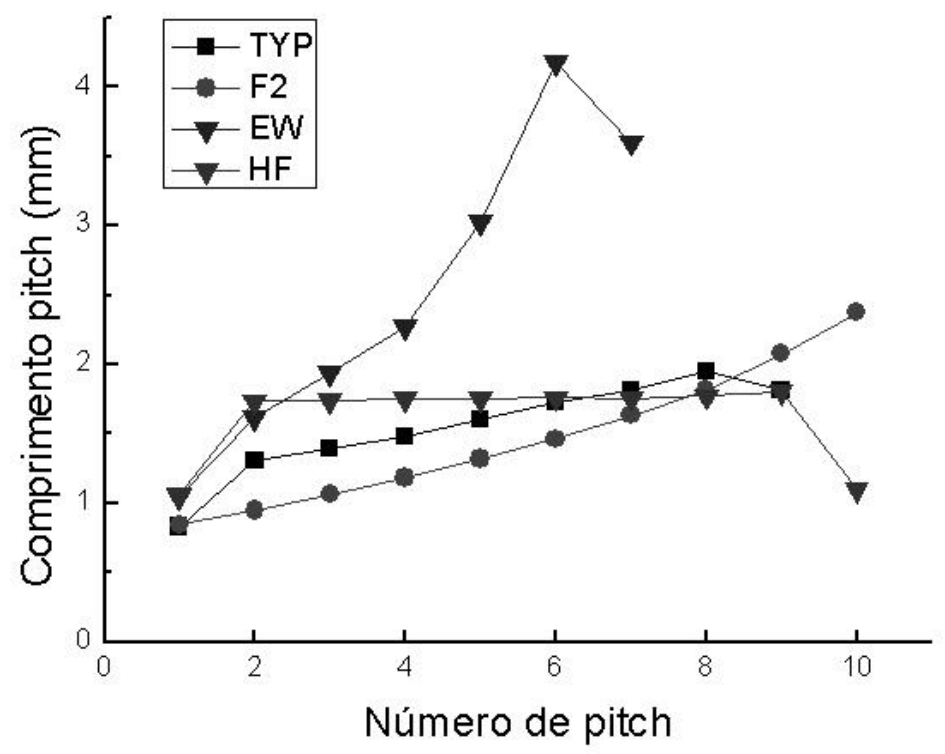

Figura 1. Valores médios de comprimentos de pitch dos instrumentos HyFlex, Typhoon, EndoWave e PTU F2 analisados.

Os valores médios e desvios-padrão da área de seção transversal a $3 \mathrm{~mm}$ da ponta (A3) dos instrumentos EW, HF, PTU F2 e TYP analisados são mostrados na Tabela 1, juntamente com os valores de momento de dobramento a $45^{\circ}$ (MD45). Pode-se observar que $\mathrm{A} 3$ dos instrumentos $\mathrm{EW}$ foi semelhante a dos instrumentos HF $(p=0,276)$. Quanto aos instrumentos PTU F2 e TYP, o primeiro apresentou A3 maior do que o último $(p=0,000)$. Os valores de MD45 para os instrumentos HF foram significativamente menores do que aqueles obtidos para os instrumentos EW $(p=0,000)$. Um resultado similar foi obtido quando os instrumentos TYP e PTU foram comparados, com o primeiro significativamente menor do que o último $(p=0,000)$.

* Contribuição técnica ao $69^{\circ}$ Congresso Anual da ABM - Internacional e ao 14ํㅡㄹ ENEMET - Encontro Nacional de Estudantes de Engenharia Metalúrgica, de Materiais e de Minas, 21 a 25 de julho de 2014, São Paulo, SP, Brasil. 


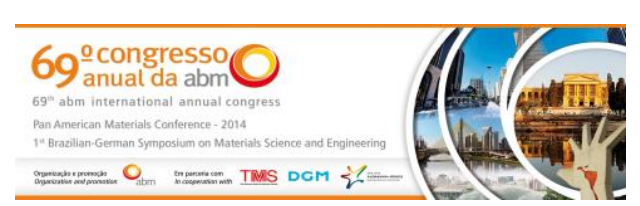

Tabela 1. Valores médios (desvio padrão) do Momento de Dobramento a $45^{\circ}$ (MD45) de instrumentos HyFlex, Typhoon, EndoWave e PTU F2

\begin{tabular}{lcc}
\hline Instrumentos & A3 $(\mathrm{mm})$ & MD45 $(\mathrm{N} . \mathrm{cm})$ \\
\hline HyFlex & $0,084(0,005)$ & $0,327(0,03)$ \\
\hline Typhoon & $0,107(0,005)$ & $0,315(0,03)$ \\
\hline EndoWave & $0,082(0,004)$ & $0,567(0,04)$ \\
\hline PTU F2 & $0,122(0,038)$ & $1,149(0,07)$ \\
\hline
\end{tabular}

Os valores de dureza medidos foram $445 \pm 20$ e $316 \pm 12 \mathrm{kgf} / \mathrm{mm}^{2}$ para EW e HF, respectivamente. Eles foram estatisticamente diferentes $(p=0,000)$, com EW mais duro do que HF. Quanto aos instrumentos PTU F2 e TYP, os valores médios encontrados foram: $381 \pm 15$ e $271 \pm 10 \mathrm{kgf} / \mathrm{mm}^{2}$, respectivamente, sendo 0 primeiro, significativamente mais duro do que o segundo $(p=0,000)$. Portanto, os instrumentos fabricados com a tecnologia CM apresentaram dureza significativamente inferior em comparação com os produzidos utilizando o NiTi convencional. Há o potencial desse resultado implicar em redução substancial da eficácia de corte dos instrumentos $\mathrm{CM}$, porque a diferença de dureza entre 0 instrumento e a dentina seria menor [21].

Os resultados dos testes de torção são mostrados na Figura 2 e indicam que a resistência à torção de instrumentos $\mathrm{EW}$ e HF, medidos em termos de torque máximo $(M T)$, foi semelhante $(p=0,228)$. A resistência à torção dos instrumentos TYP foi significativamente menor do que os instrumentos PTU F2 ( $p=0,034)$.

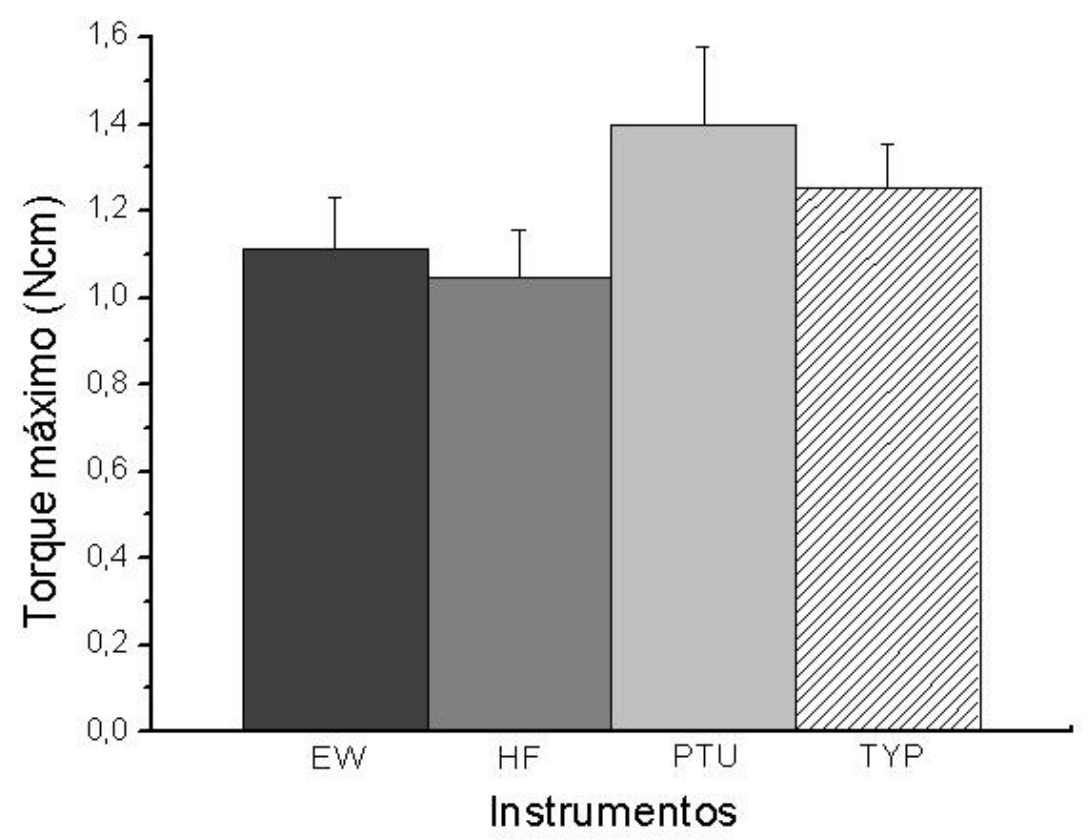

Figura 2. Valores médios de torque máximo de instrumentos HyFlex, Typhoon, EndoWave e PTU F2 ensaiados em torção até a ruptura.

Em geral, comprimento de pitch menor tende a proporcionar maior resistência à torção ao instrumento e reduz a eficiência de corte, ao passo que um comprimento de pitch maior tende a permitir uma maior eficiência de corte e uma remoção mais eficiente dos detritos, além de prevenir o emparafusamento no canal

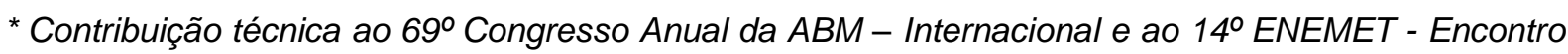
Nacional de Estudantes de Engenharia Metalúrgica, de Materiais e de Minas, 21 a 25 de julho de 2014, São Paulo, SP, Brasil.
} 
radicular [19,20]. Embora os instrumentos EW apresentarem comprimentos de pitch maiores do que aqueles apresentados pelos instrumentos HF, o MD encontrado para os últimos foi significativamente mais baixo do que para $\mathrm{EW}$, o que, provavelmente, resulta de uma maior flexibilidade da tecnologia CM. Seria de se esperar que os instrumentos EW apresentassem valores médios mais baixos de torque em comparação com instrumentos HF, o que não foi confirmado pelo teste de torção. Instrumentos PTU F2 e TYP apresentaram crescimento contínuo de comprimentos de pitch, o que provavelmente não interfere em suas propriedades mecânicas.

Quando as propriedades mecânicas de instrumentos superelásticos convencionais de $\mathrm{NiTi}$ e aqueles fabricados com a tecnologia $\mathrm{CM}$ de áreas transversais semelhantes foram comparados, como por exemplo EW e HF, a influência do método de fabricação pode ser melhor apreciada. Não houve diferença significativa nos valores de $A 3$ encontrados nos instrumentos $E W$ e HF, portanto esses fatores não podem ter influenciado seu comportamento mecânico. Já os instrumentos PTU F2 apresentaram A3 significativamente maior do que os TYP, o que pode ter determinado a flexibilidade mais baixa exibida pelos primeiros, mas possivelmente a tecnologia CM utilizada na fabricação dos instrumentos TYP teve papel decisivo nesse comportamento.

Os resultados do presente estudo mostraram que os instrumentos CM foram os instrumentos mais flexíveis, com uma melhora significativa na flexibilidade sobre os outros instrumentos testados (Tabela 1). Apesar do fato de que a flexibilidade é influenciada pelo design do instrumento, uma grande melhoria provavelmente está relacionada com o processo de fabricação dos instrumentos CM (que não é divulgado pelo fabricante), porque eles possuem um desenho de seção transversal muito semelhante aos dos outros instrumentos testados. Os resultados obtidos estão em acordo os relatados por outros autores [15,16,22], que encontraram instrumentos $\mathrm{CM}$ mais flexíveis do que outros instrumentos rotatórios fabricados com fios de $\mathrm{NiTi}$ superelásticos. No entanto, esses autores não avaliaram as características geométricas dos instrumentos testados, o que pode ter influenciado os resultados. Sabe-se que tratamentos termomecânicos de ligas NiTi têm um forte impacto sobre o seu comportamento, particularmente no que diz respeito às temperaturas de transformação martensítica e reversa [23]. É possível que os tratamentos utilizados durante a fabricação dos instrumentos CM possam facilitar a ocorrência da fase $R$ induzida por tensão $[9,24]$, reduzindo, assim, o módulo de elasticidade aparente da austenita e a tensão de transformação do material.

A resistência torcional dos instrumentos $\mathrm{CM}$ em relação a outros instrumentos foi estudada por diversos autores [14,16,17], que verificaram forte dependência dessa propriedade com a marca, tamanho e conicidade dos mesmos. Em um desses estudos [14], verificou-se que os instrumentos HF apresentam resistência à torção semelhante aos instrumentos fabricados com NiTi convencional. As conclusões desse trabalho são consistentes com os resultados apresentados por EW e HF no presente estudo. No entanto, em nenhum dos trabalhos mencionados a área da seção transversal dos instrumentos foi considerada, o que pode ter influenciado os resultados. No caso de PTU F2, a sua maior resistência à torção em relação aos instrumentos TYP pode ser atribuída à sua área em corte transversal significativamente superior a $3 \mathrm{~mm}$ da ponta, posição em que as tensões de cisalhamento se concentram, devido à conicidade dos instrumentos e sua apreensão nessa posição durante os ensaios.

\footnotetext{
* Contribuição técnica ao $69^{\circ}$ Congresso Anual da ABM - Internacional e ao 14ํㅡㄹ ENEMET - Encontro Nacional de Estudantes de Engenharia Metalúrgica, de Materiais e de Minas, 21 a 25 de julho de 2014, São Paulo, SP, Brasil.
} 


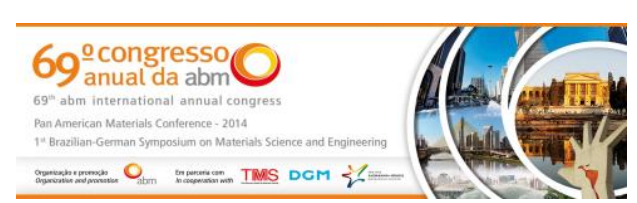

\section{CONCLUSÃo}

Os resultados encontrados mostraram que a tecnologia CM permite a produção de instrumentos endodônticos mais flexíveis, o que é desejável do ponto de vista clínico, porém sem comprometer sua resistência torcional.

\section{Agradecimentos}

Os autores agradecem à Capes, CNPq e FAPEMIG por suporte financeiro para realização do trabalho de pesquisa.

\section{REFERÊNCIAS}

1 Walia $\mathrm{H}$, Brantley WA, Gerstein $\mathrm{H}$. An initial investigation of the bending and torsional properties of nitinol root canal files. J Endod 1988; 14: 346-51.

2 Glosson CR, Haller RH, Dove SB, et al. A comparison of root canal preparations using $\mathrm{Ni}-\mathrm{Ti}$ hand, $\mathrm{Ni}$-Ti engine-driven, and K-Flex endodontic instruments. J Endod 1995; 21: 146-51.

3 Thompson SA. An overview of nickel-titanium alloys used in dentistry. Int Endod J 2000; 33: 297-310.

4 Alapati SB, Brantley WA, lijima M, et al. Metallurgical characterization of a new nickeltitanium wire for rotary endodontic instruments. J Endod 2009; 35:1589-93.

5 Johnson E, Lloyd A, Kuttler S, et al. Comparison between a novel nickel titanium alloy and 508 nitinol on the cyclic fatigue life of ProFile 25/.04 rotary instruments. J Endod 2008; 34:1406-9.

6 Shen Y, Zhou H, Zheng Y, Peng B, Haapasalo M. Current Challenges and Concepts of the Thermomechanical Treatment of Nickel-Titanium Instruments. J Endod, 2013; 39: 163-72.

7 Larsen CM, Watanabe I, Glickman G, He J. Cyclic fatigue analysis of a new generation of nickel titanium rotary instruments. J Endod, 2009; 35: 401-3.

8 Peixoto IFC, Pereira ESJ, Silva JG, Viana ACD, Buono VTL, Bahia MGA. Flexural fatigue and torsional resistance of ProFile GT and ProFile GT series $X$ instruments. $J$ Endod 2010; 36: 741-4.

9 Pereira ESJ, Peixoto IFC, Viana ACD et al. Physical and mechanical properties of a thermomechanically treated $\mathrm{NiTi}$ wire used in the manufacture of rotary endodontic instruments. Int Endod J 2012; 45: 469-74.

10 CM Wire press release. Johnson City, TN: DS Dental; 2010.

11 Shen Y, Qian W, Abtin H, Gao Y, Haapasalo M. Fatigue testing of controlled memory wire nickel-titanium rotary instruments. J Endod 2011a; 37: 997-1001.

12 Shen Y, Zhou H-M, Zheng Y-F, Campbell L, Peng B, Haapasalo M. Metallurgical characterization of controlled memory wire nickel-titanium rotary instruments. J Endod 2011b; 37: 1566-71.

13 Shen Y, Qian W, Abtin H, et al. Effect of environment on fatigue failure of controlled memory wire nickel-titanium rotary instruments. J Endod 2012;38: 376-80.

14 Peters OA, Gluskin AK,Weiss RA, et al. An in vitro assessment of the physical properties of novel Hyflex nickel-titanium rotary instruments. Int Endod J 2012; 45: 1027-34.

15 Testarelli L, Plotino G, Al-Sudani D, et al. Bending properties of a new nickel-titanium alloy with a lower percent by weight of nickel. J Endod 2011; 37: 1293-5.

16 Ninan, E. and D.W. Berzins. Torsion and bending properties of shape memory and superelastic nickel-titanium rotary instruments. J Endod 2013; 39: 101-4.

\footnotetext{
* Contribuição técnica ao $69^{\circ}$ Congresso Anual da ABM - Internacional e ao 14ํㅡㄹ ENEMET - Encontro Nacional de Estudantes de Engenharia Metalúrgica, de Materiais e de Minas, 21 a 25 de julho de 2014, São Paulo, SP, Brasil.
} 
